Publ. RIMS, Kyoto Univ.

18 (1982), 265-282

\title{
Pursell-Shanks Type Theorem for Orbit Spaces of G-Manifolds
}

\author{
By
}

Kōjun $\mathrm{ABE}^{*}$

\section{$\S 0 . \quad$ Introduction}

Pursell and Shanks [8] proved that a Lie algebra isomorphism between Lie algebras of all $C^{\infty}$ vector fields with compact support on paracompact connected $C^{\infty}$ manifolds $M$ and $N$ yields a diffeomorphism between the manifolds $M$ and $N$. Similar results hold for some other structures on manifolds. Indeed, Omori [6] proved the corresponding results in the case of volume structures, symplectic structures, contact structures and fibering structures with compact fibers. The case of complex structures was studied by Amemiya [1]. Koriyama [5] proved that in the case of Lie algebras of vector fields with invariant submanifolds.

Recently, Fukui [4] studies the case of Lie algebras of $G$-invariant $C^{\infty}$ vector fields with compact support on paracompact free smooth $G$ manifolds when $G$ is a compact connected semi-simple Lie group. The corresponding result is no longer true when $G$ is not semi-simple or $G$ does not act freely.

In this paper, we consider Pursell-Shanks type theorem for orbit spaces of smooth $G$-manifolds in the case of $G$ a compact Lie group. For a smooth $G$-manifold $M$, the orbit space $M / G$ inherits a smooth structure by defining a function on $M / G$ to be smooth if it pulls back to a smooth function on $M$, and the Zariski tangent space of $M / G$ can be defined. This smooth structure of the orbit space was studied by Schwarz [9], [11], Bierstone [2], Poénaru [7] and Davis [3]. Schwarz [10] defined a Lie algebra $\mathfrak{X}(M / G)$ of smooth vector fields on the orbit

Communicated by N. Shimada, April 20, 1981.

* Department of Mathematics, Faculty of Liberal Arts, Shinshu University, Matsumoto, 390, Japan. 
space $M / G$, and proved $\pi_{*}\left(\mathfrak{x}_{G}(M)\right)=\mathfrak{X}(M / G)$, where $\mathfrak{X}_{G}(M)$ is the Lie algebra of all $G$-invariant $C^{\infty}$ vector fields with compact support on $M$ and $\pi: M \rightarrow M / G$ is a natural projection.

The purpose of this paper is to prove the following:

Theorem. Let $G$ and $G^{\prime}$ be compact Lie groups. Let $M$ and $N$ be connected paracompact smooth $G$-manifold and $G^{\prime}$-manifold without boundary, respectively. There exists a Lie algebra isomorphism $\Phi: \mathfrak{X}(M / G) \rightarrow \mathfrak{X}\left(N / G^{\prime}\right)$ if and only if there exists a strata preserving diffeomorphism $\sigma: M / G \rightarrow N / G^{\prime}$ such that $\Phi=\sigma_{*}$.

Main part of the proof of our theorem is to find maximal ideals of $\mathfrak{X}(M / G)$. By the theorem of Schwarz, maximal ideals of $\mathfrak{X}(M / G)$ are induced from those of $\mathfrak{X}_{G}(M)$. To determine the maximal ideals of $\mathfrak{X}_{G}(M)$, we use the parallel method to those of Pursell-Shanks [8] and Koriyama [5].

\section{$\S 1$. The Tangent Space of an Orbit Space}

In this paper, we consider $C^{\infty}$ smooth category. Let $G$ and $G^{\prime}$ be compact Lie groups. Let $M$ and $N$ be connected paracompact smooth $G$-manifold and $G^{\prime}$-manifold without boundary, respectively. Put $\bar{M}$ $=M / G, \bar{N}=N / G^{\prime}$. The orbit space $\bar{M}$ has an induced smooth structure such that a function $f: \bar{M} \rightarrow R$ is smooth if the composition $M \stackrel{\pi}{\rightarrow} \bar{M} \stackrel{f}{\rightarrow} R$ is smooth, where $\pi$ is the natural projection. Let $C^{\infty}(\bar{M})$ denote the set of all smooth functions on $\bar{M}$. A map $h: \bar{M} \rightarrow \bar{N}$ is smooth if, $f \circ h \in C^{\infty}(\bar{M})$ for any $f \in C^{\infty}(\bar{N})$, and we say that $h$ is diffeomorphic if $h^{-1}$ is also smooth.

We can define a tangent space of the orbit space as usual. A tangent vector $v$ of $\bar{M}$ at $p$ is a correspondense assigning to any smooth functions $f, g$ around $p$ real numbers $v(f), v(g)$ with the following conditions :

(1) $v(\lambda f+\mu g)=\lambda v(f)+\mu v(g)$ for $\lambda, \mu \in R$,

(2) $\quad v(f g)=v(f) g(p)+f(p) v(g)$.

Put $\tau(\bar{M})=\bigcup_{p \in \bar{M}} \tau_{p}(\bar{M})$. 
Given $a \in M$, let $G_{a}$ denote the isotropy group at $a$ and $V_{a}$ be a linear slice at $a$. Then $V_{a}$ is a $G_{a}$-module. Put $p=\pi(a)$ and $\bar{V}_{p}=V_{a} / G_{a}$. Then $\bar{V}_{p}$ is an open neighborhood of $p$ in $\bar{M}$.

Proposition 1.1 (cf. Davis [3], Proposition 2.3).

(1) $\tau_{p}(\bar{M}) \cong \tau_{p}\left(\bar{V}_{p}\right)$.

(2) Let $\bar{M}_{p}$ denote the germs of smooth functions on $\bar{V}_{p}$ which vanish at $p$. Then $\tau_{p}\left(\bar{V}_{p}\right) \cong \operatorname{Hom}\left(\overline{\mathfrak{M}}_{p} / \overline{\mathfrak{M}}_{p}{ }^{2}, R\right)$.

Let $H$ be a compact Lie group and let $\mathrm{V}$ be an $\mathrm{H}$-module. By a theorem of Hilbert ([13], p. 275), the algebra of $H$-invariant polynomials $R[V]^{H}$ is finitely gencrated.

Theorem 1.2 (Schwarz [9]). Let $\left\{\theta_{1}, \cdots, \theta_{s}\right\}$ be a set of generators for $R[V]^{H}$, and let $\theta=\left(\theta_{1}, \cdots, \theta_{s}\right): V \rightarrow R^{s}$. Then

(1) $\theta^{*} C^{\infty}\left(R^{s}\right)=C_{H}^{\infty}(V)$.

(2) The orbit map $\bar{\theta}: V / H \rightarrow R^{s}$ of $\theta$ is a topological embedding.

Proposition 1.3 (cf. Davis [3] Lemma 2.1). Let $R[V]_{0}^{H}$ denote the algebra of $H$-invariant polynomials rehich vanish at 0 . Then

(1) $\overline{\mathfrak{M}}_{0} / \overline{\mathfrak{M}}_{0}^{2} \cong R[V]_{0}^{H} /\left(R[V]_{0}^{H}\right)^{2}$.

(2) If $\left\{\theta_{1}, \cdots, \theta_{s}\right\}$ is a minimal set of generators for $R[V]_{0}^{H}$, then the dimension of $\tau_{0}(V / H)$ is $s$.

\section{§ 2. Smooth Vector Fields on an Orbit Space}

Let $X: \bar{M} \rightarrow \tau(\bar{M})$ be a section. For any $f \in C^{\infty}(\bar{M})$, we can define a function $X(f): \bar{M} \rightarrow R$ by $X(f)(p)=X_{p}(f)$. If $X(f) \in$ $C^{\infty}(\bar{M})$ for any $f \in C^{\infty}(\bar{M})$, then we say $Y$ is a smooth vector field on $\bar{M}$. Let $\mathfrak{D}(\bar{M})$ denote the Lie algebra of all smooth vector fields on $\bar{M}$. Let $D C^{\infty}(\bar{M})$ denote the set of all derivations of $C^{\infty}(\bar{M})$. Using Theorem 1.2 (1) we have:

Proposition 2.1. $\mathfrak{D}(\bar{M})$ is isomorphic to $D C^{\infty}(\bar{M})$ as a Lie algebra. 
The orbit space $\bar{M}$ is stratified by its orbit type.

Definition 2.2 (Schwarz [11]). A smooth vector field $X$ on $\bar{M}$ is said to be strata preserving if $X_{p} \in \tau_{p}\left(\sigma_{p}\right)$ for any $p \in \bar{M}$, where $\sigma_{p}$ denotes the stratum of $\bar{M}$ containing $p$. Let $\mathfrak{X}(\bar{M})$ denote the set of all strata preserving smooth vector fields with compact support on $\bar{M}$. $\mathfrak{X}(\bar{M})$ is a Lie subalgebra of $D C^{\infty}(\bar{M})$. Let $\mathfrak{X}_{G}(M)$ denote the set of all $G$-invariant smooth vector fields with compact support on $M$. There is a Lie algebra homomorphism $\pi_{*}: \mathfrak{X}_{G}(M) \rightarrow D C^{\infty}(\bar{M})$ defined by $\pi_{*}(X)(\bar{f})$ $=X(f)$, where $f \in C_{G}^{\infty}(M)$ and $\bar{f}$ is the orbit map of $f$.

Theorem 2.3 (Schwarz [11]). The image of the homomorphism $\pi_{*}: \mathfrak{X}_{G}(M) \rightarrow D C^{\infty}(\bar{M})$ is $\mathfrak{X}(\bar{M})$.

\section{§ 3. Maximal Ideals of $\mathfrak{\mathfrak { X }}(\bar{M})$}

Let $a \in M$ and put $p=\pi(a) \in \bar{M}$. Let $V_{a}$ be a linear slice at $a$. Then $N_{a}=G \times{ }_{a_{a}} V_{a}$ is equivalent to a linear tubular neighborhood of the orbit $G(a)$ of $a$. Let $\tau\left(N_{a}\right)$ be the tangent bundle of the $G$-manifold $N_{a}$, and let $\Gamma_{G}\left(\tau\left(N_{a}\right)\right)$ denote the set of all $G$-invariant smooth sections of $\tau\left(N_{a}\right)$. Let $\tau\left(V_{a}\right)$ be the tangent bundle of the $G_{a}$-manifold $V_{a}$, and let $\Gamma_{G_{a}}\left(\tau\left(V_{a}\right)\right)$ denote the set of all $G_{a}$-invariant smooth sections of $\tau\left(V_{a}\right)$. Then we have canonical isomorphisms $\Gamma_{G}\left(\tau\left(N_{a}\right)\right) \cong \Gamma_{G_{a}}\left(\tau\left(N_{a}\right) \mid V_{a}\right)$ and $C_{G}^{\infty}\left(N_{a}\right) \cong C_{a_{a}}^{\infty}\left(V_{a}\right)$. It is easy to see the following:

Lemma 3.1. (1) For any $X \in \Gamma_{a_{a}}\left(\tau\left(V_{a}\right)\right)$, there exists $Y \in$ $\mathfrak{x}_{G}(M)$ such that $Y=X$ on a $G_{a}$-invariant neighborhood $U_{a}$ of $a$ in $V_{a}$.

(2) For any $f \in C_{G_{a}}^{\infty}\left(V_{a}\right)$, there exists $F \in C_{G}^{\infty}(M)$ such that $F=f$ on a $G_{a}$-invariant neighborhood $U_{a}$ of $a$ in $V_{a}$.

Put $\bar{M}_{0}=\left\{q \in \bar{M} ; X_{q}=0\right.$ for any $\left.X \in \mathfrak{X}(\bar{M})\right\}$, and put $\bar{M}_{1}=\bar{M}-\bar{M}_{0}$.

Proposition 3.2. $\bar{M}_{0}$ is discrete.

Proof. For any $a \in M$, let $\left\{x_{1}, \cdots, x_{n}\right\}$ be a canonical coordinate of a 
linear slice $V_{a}$ of $a$. We can assume $G_{a}$ acts orthogonally on $V_{a}$. Then the radial vector field $X=\sum_{i=1}^{n} x_{i} \frac{\partial}{\partial x_{i}}$ is a $G_{a}$-invariant smooth vector field on $V_{a}$. Let $f: V_{a} \rightarrow R$ be a $G_{a}$-invariant smooth function defined by $f\left(x_{1}, \cdots, x_{n}\right)=x_{1}{ }^{2}+\cdots+x_{n}{ }^{2}$. By Lemma 3.1, there exist $Y \in \mathfrak{X}_{G}(M)$ and $F \in C_{G}^{\infty}(M)$ such that $Y=X$ and $F=f$ on a $G_{a}$-invariant neighborhood $U_{a}$ of a in $V_{a}$, respectively. Put $\bar{U}_{p}=U_{a} / G_{a}, \bar{Y}=\pi_{*}(Y)$ and let $\bar{F}$ be the orbit map of $F$. Then $\bar{Y}(\bar{F}) \neq 0$ on $\bar{U}_{p}-\{p\}$, and Proposition 3.2 follows.

Note that $\pi_{a}: N_{a} \rightarrow G(a)$ is a $G$-vector bundle. The tangent bundle $\tau\left(N_{a}\right)$ of $N_{a}$ is isomorphic to $\pi_{a}^{*}\left(\tau(G(a)) \oplus \xi_{a}\right.$ as a $G$-vector bundle, where $\xi_{a}$ is a bundle along the fibres of $N_{a}$. Let $r_{a}$ be the composition

$$
\begin{aligned}
& \mathfrak{x}_{G}(M) \stackrel{\text { restriction }}{\longrightarrow} \Gamma_{G}\left(\tau\left(N_{a}\right)\right) \cong \Gamma_{G_{a}}\left(\tau\left(N_{a}\right) \mid V_{a}\right) \\
& \stackrel{\text { projection }}{\longrightarrow} \Gamma_{G_{a}}\left(\xi_{a} \mid V_{a}\right) \cong \Gamma_{G_{a}}\left(\tau\left(V_{a}\right)\right) .
\end{aligned}
$$

It is easy to see that $r_{a}$ is a Lie algebra homomorphism. Put $\Gamma_{G_{a}}\left(\tau\left(V_{a}\right)\right)_{0}$ $=\left\{X \in \Gamma_{G_{a}}\left(\tau\left(V_{a}\right)\right) ; X_{a}=0\right\}$. For $X \in \Gamma_{a_{a}}\left(\tau\left(V_{a}\right)\right)_{0}$, we denote $j_{a}^{r}(X)$ the $r$-jet of $X$ at $a(r=1,2, \cdots)$. Put $\Gamma_{G_{a}}\left(\tau\left(V_{a}\right)\right)_{0}^{k}=\left\{X \in \Gamma_{G_{a}}\left(\tau\left(V_{a}\right)\right)_{0}\right.$; $j_{a}^{r}(X)=0$ for $\left.1 \leqq r \leqq k\right\} \quad(1 \leqq k \leqq \infty)$.

For $q \in \bar{M}_{0}$, choose a point $b \in \pi^{-1}(q)$. Let $\mathrm{gl}_{G_{b}}\left(V_{b}\right)$ denote the set of $G_{b}$-invariant endomorphisms of $V_{b}$. Note that, for $X \in \Gamma\left(\tau\left(V_{b}\right)\right), j_{b}^{1}(X)$ defines an element of $\mathfrak{g l}\left(V_{b}\right)$ as usual. It is easy to see that $j_{b}^{1}(X) \in$ $\mathrm{gl}_{G_{b}}\left(V_{b}\right)_{b}$ for $X \in \Gamma_{G_{b}}\left(\tau\left(V_{b}\right)\right)$.

Lemma 3. 3. $j_{b}^{1}: \Gamma_{G_{b}}\left(\tau\left(V_{b}\right)\right) \rightarrow \mathfrak{g} \int_{G_{b}}\left(V_{b}\right)$ is an onto Lie algebra homomorphism.

Proof. Since $\pi(b)=q \in \bar{M}_{0}, \Gamma_{G_{b}}\left(\tau\left(V_{b}\right)\right)=\Gamma_{G_{b}}\left(\tau\left(V_{b}\right)\right)_{0}$. Then $\Gamma_{G_{b}}(\tau$ $\left.\left(V_{b}\right)\right) / \Gamma_{G_{b}}\left(\tau\left(V_{b}\right)\right)_{0}^{1} \cong \mathrm{gl}_{G_{b}}\left(V_{b}\right)$ and Lemma 3.3 follows.

By Proposition 3.2, $\bar{M}_{0}$ is discrete. Then $\bar{M}_{0}$ is a countable set $\left\{q_{i} ; i \in I\right\}$. Choose a point $b_{i} \in \pi^{-1}\left(q_{i}\right)$ for each $q_{i} \in \bar{M}_{0}$. Put $J_{i}^{1}(M)$ $=\mathfrak{g l} l_{b_{b_{i}}}\left(V_{b_{i}}\right)$ and put $J^{1}(M)=\prod_{i \in I} J_{i}^{1}(M)$ which consists of those elements having only finite number of non-zero factors. Then we have: 
Corollary 3.4. The composition

$$
J^{1}: \mathfrak{X}_{G}(M) \stackrel{\prod_{i \in I} r_{i}}{\longrightarrow} \prod_{i \in I} \Gamma_{G_{b_{i}}}\left(\tau\left(V_{b_{i}}\right)\right) \stackrel{\prod_{i \in I} j_{b_{i}}^{1}}{\longrightarrow} J^{1}(M)
$$

is an onto Lie algebra homomorphism.

$G_{b_{i}}$-module $V_{b_{i}}$ is isomorphic to $\bigoplus_{j} d_{i j} W_{i j}$. Here $d_{i j}$ is a non-negative integer and $W_{i j}$ runs over the inequivalent irreducible $G_{b_{i}}$-modules. Let $K_{i j}$ be the real numbers $R$, complex numbers $C$ or quaternionic numbers $H$ if $\operatorname{dim}_{R} \mathfrak{g l}_{G_{b_{i}}}\left(W_{i j}\right)=1,2$ or 4 , respectively. Then $\mathfrak{g} \mathfrak{l}_{G_{b_{i}}}\left(V_{b_{i}}\right) \cong \underset{j}{\bigoplus} \mathfrak{g l}\left(d_{i j}\right.$, $\left.K_{i j}\right)$.

Proposition 3.5. $\mathfrak{g l}(d, R) \cong R \oplus \mathfrak{I l}(d, R)$,

$$
\begin{aligned}
& \mathfrak{g l}(d, C) \cong C \oplus \mathfrak{I l}(d, C) \text { and } \\
& \mathfrak{g l}(d, H) \cong R \oplus \mathfrak{I l}(d, H),
\end{aligned}
$$

where $\mathfrak{g l}(d, K)=[\mathfrak{g l}(d, K), \mathfrak{g l}(d, K)]$ for $K=R, C$ or $H$.

Proof. Note that $\mathfrak{g l}(d, H)=\{X \in \mathfrak{g l}(n, H) ; \operatorname{Re} \operatorname{Tr}(X)=0\} \quad$ and $\mathfrak{g} \mathfrak{l}(d, H)$ is a simple Lie algebra. Other cases are similar to this.

Next we consider maximal ideals of $\Gamma_{G_{a}}\left(\tau\left(V_{a}\right)\right)$ for $a \in M$ such that $\pi(a)=p \in \bar{M}_{1}$. First we need the following:

Lemma 3. 6. Let $H$ be a compact Lie group and let $V$ be an $H$-module. For $Y \in \Gamma(\tau(V))$, we define $\widetilde{Y} \in \Gamma_{H}(\tau(V))$ by $\widetilde{Y}_{p}=\int_{H}\left(h_{*} Y\right)_{p}$ $d h$ for $p \in V$. Then $[X, \widetilde{Y}]=\int_{H} h_{*}[X, Y] d h$ for $X \in \Gamma_{H}(\tau(V))$. Here $\left(h_{*} Y\right)_{p}=(d h)_{h-1 p} Y_{h^{-1} p}$.

Proof. Let $\left\{x_{1}, \cdots, x_{n}\right\}$ be a canonical coordinate of $V$. For $p \in V$, $f \in C^{\infty}(V)$, we have:

$$
\begin{aligned}
\widetilde{Y}_{p}(f) & =\left(\int_{H}\left(\sum_{i=1}^{n}\left(h_{*} Y\right)_{p}\left(x_{i}\right)\left(\frac{\partial}{\partial x_{i}}\right)_{p}\right) d h\right)(f) \\
& =\sum_{i=1}^{n}\left(\int_{H}\left(h_{*} Y\right)_{p}\left(x_{i}\right) d h\right)\left(\frac{\partial}{\partial x_{i}}\right)_{p}(f)
\end{aligned}
$$




$$
\begin{aligned}
& =\int_{H}\left(\sum_{i=1}^{n}\left(h_{*} Y\right)_{p}\left(x_{i}\right)\left(\frac{\partial f}{\partial x_{i}}\right)_{p}\right) d h \\
& =\int_{H}\left(h_{*} Y\right)_{p}(f) d h
\end{aligned}
$$

Then $[X, \widetilde{Y}]_{p}(f)=X_{p}\left(\int_{H}\left(h_{*} Y\right)(f) d h\right)-\int_{H}\left(h_{*} Y\right)_{p}(X f) d h$

$$
\begin{aligned}
& =\int_{H}\left(X_{p}\left(h_{*} Y\right)(f)-\left(h_{*} Y\right)_{p}(X f)\right) d h \\
& =\int_{H}\left[X, h_{*} Y\right]_{p}(f) d h \\
& =\left(\int_{H}\left[X, h_{*} Y\right]_{p} d h\right)(f) .
\end{aligned}
$$

Lemma 3. 7. Suppose that $\mathfrak{N}$ is a proper ideal of $\Gamma_{G_{a}}\left(\tau\left(V_{a}\right)\right)$ which contains $\Gamma_{G_{a}}\left(\tau\left(V_{a}\right)\right)_{0}{ }^{\infty}$ for $a \in M$ such that $\pi(a)=p \in \bar{M}_{1}$. Then $\mathfrak{N}$ is contained in $\Gamma_{G_{a}}\left(\tau\left(V_{a}\right)\right)_{0}$.

Proof. Suppose there exists $X \in \mathfrak{N}$ with $X_{a} \neq 0$. By Koriyama [5] Lemma 2.1, for any $Z \in \Gamma_{G_{a}}\left(\tau\left(V_{a}\right)\right)$ there exist a $G_{a}$-invariant neighborhood $U$ of $a$ in $V_{a}$ and $Y \in \Gamma\left(\tau\left(V_{a}\right)\right)$ such that $[X, Y]=Z$ on $U$. Put $\widetilde{Y}=\int_{G_{a}} g_{*} Y d g \in \Gamma_{a_{a}}\left(\tau\left(V_{a}\right)\right)$. By Lemma 3.6 , we have $[X, \widetilde{Y}]=$ $\int_{G_{a}} g_{*}[X, Y] d g=\int_{G_{a}} g_{*} Z d g=Z$ on $U$. Put $Z_{1}=Z-[X, \widetilde{Y}]$. Then $Z_{1} \in$ $\Gamma_{G_{a}}\left(\tau\left(V_{a}\right)\right)_{0}^{\infty}$ which is contained in $\mathfrak{N}$. Since $\mathfrak{N}$ is an ideal, $Z \in \mathfrak{R}$. Thus $\mathfrak{N}=\Gamma_{G_{a}}\left(\tau\left(V_{a}\right)\right)$ which is a contradiction to $\mathfrak{N}$ a proper ideal.

By Lemma 3. 7, there exists a unique maximal ideal $\mathfrak{N}_{a}$ of $\Gamma_{G_{a}}\left(\tau\left(V_{a}\right)\right)$ satisfying $\Gamma_{G_{a}}\left(\tau\left(V_{a}\right)\right)_{0}{ }^{\infty} \subset \mathfrak{N}_{a} \subset \Gamma_{G_{a}}\left(\tau\left(V_{a}\right)\right)_{0}$. Put $\Im_{a}=\left\{X \in \mathfrak{X}_{G}(M) ; r_{a}(X)\right.$ $\left.\in \mathfrak{l}_{a}\right\}$

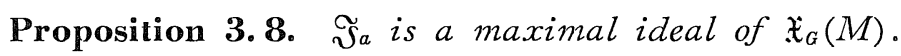

Proof. Put $V_{a}(\rho)=\left\{v \in V_{a} ;\|v\|<\rho\right\}$ for a positive number $\rho . \quad \mathfrak{l}_{a}(\rho)$ $=\left\{Y \in \Gamma_{a_{a}}\left(\tau\left(V_{a}\right)\right)\right.$; supp $\left.Y \subset V_{a}-V_{a}(\rho)\right\}$ is an ideal of $\Gamma_{a_{a}}\left(\tau\left(V_{a}\right)\right)$ which is contained in $\Gamma_{G_{a}}\left(\tau\left(V_{a}\right)\right)_{0}$. Then $\Re_{a}(\rho)$ is contained in $\Re_{a}$. It is clear that $\mathfrak{\Im}_{a}$ is an ideal of $\mathfrak{X}_{G}(M)$. 
Let $\mathfrak{M}$ be a maximal ideal of $\mathfrak{X}_{a}(M)$ which contains $\mathfrak{\Im}_{a}$. Suppose that there exists $X \in \mathfrak{M}$ with $r_{a}(X)_{a} \neq 0$. Similarly as in the proof of Lemma 3.7 , we can prove $\mathfrak{M}=\mathfrak{X}_{G}(M)$, which is a contradiction. Then $r_{a}(\mathfrak{M})$ is contained in $\Gamma_{G_{a}}\left(\tau\left(V_{a}\right)\right)_{0}$. Combining $\mathfrak{N}_{a}(\rho) \subset \mathfrak{N}_{a}$ and Lemma 3.1, we see $r_{a}(\mathfrak{M})+\mathfrak{N}_{a}$ is an ideal of $\Gamma_{G_{a}}\left(\tau\left(V_{a}\right)\right)$. Therefore $r_{a}(\mathfrak{M})$ is contained in $\mathfrak{N}_{a}$, and $\mathfrak{M}=\mathfrak{J}_{a}$. Thus Proposition 3.8 follows.

Put $\quad \overline{\mathfrak{\Im}}_{p}=\pi_{*}\left(\widetilde{\Im}_{a}\right)$ and $\mathfrak{X}(\bar{M})_{p}=\left\{X \in \mathfrak{X}(\bar{M}) ; X_{p}=0\right\}$. Then $\overline{\widetilde{\Im}}_{p}$ is contained in $\mathfrak{X}(\bar{M})_{p}$, and $\overline{\mathfrak{\Im}}_{p}$ is a maximal ideal.

Lemma 3.9. (1) $\bar{\Im}_{p}$ is an infinite codimensional maximal ideal of $\mathfrak{X}(\bar{M})$ for $p \in \bar{M}_{1}$.

(2) For a maximal ideal \& of $J^{1}(M)$, put $\mathfrak{M}=\left(J^{1}\right)^{-1}(\mathfrak{Q})$. Then $\mathfrak{M}$ is a finite codimensional maximal ideal of $\mathfrak{X}_{G}(M)$.

Proof. (1) For $a \in \pi^{-1}(p)$, there exists $X \in \Gamma_{G_{a}}\left(\tau\left(V_{a}\right)\right)$ with $X_{a}$ $\neq 0$. Then there exists a $G_{a}$-invariant local one parameter group of transformations $\phi ;(-\varepsilon, \varepsilon) \times U \rightarrow V_{a}$ defined on a $G_{a}$-invariant neighborhood $U$ such that $\frac{\partial \phi}{\partial t}(t, u)=X_{\phi(t, u)}$. Let $\theta:(-\varepsilon, \varepsilon) \rightarrow V_{a}$ be a map defined by $\theta(t)=\phi(t, a)$. Since $X_{a} \neq 0, \theta$ is an embedding for a sufficiently small number $\varepsilon$. Let $W$ be a $G_{a}$-invariant normal space of $\theta((-\varepsilon, \varepsilon))$ at $a$ in $V_{a}$. Then we may assume that $\phi:(-\varepsilon, \varepsilon) \times W \rightarrow V_{a}$ is a $G_{a}$-invariant embedding. Let $\left\{w_{1}, \cdots, w_{n-1}\right\}$ be a canonical coordinate of $W$. We have a local coordinate $\left\{x_{1}, \cdots, x_{n}\right\}$ of $V_{a}$ around a neighborhood $U_{1}=\phi((-\varepsilon, \varepsilon) \times W)$ of $a$ given by $x_{1}\left(\phi\left(t, w_{1}, \cdots, w_{n-1}\right)\right)=t, x_{i}\left(\phi\left(t, w_{1}\right.\right.$, $\left.\left.\cdots, w_{n-1}\right)\right)=w_{i-1}$ for $i=2, \cdots, n$. Note that $X=\frac{\partial}{\partial x_{1}}$ on $U_{1}$.

By Lemma 3.1. there are $X_{1} \in \mathfrak{X}_{G}(M)$ and $f \in C_{G}^{\infty}(M)$ such that $X_{1}=X$ and $f=x_{1}$ on a neighborhood $U_{2} \subset U_{1}$ of $a$ in $V_{a}$, respectively. Let $Y \in \Im_{a}$ and $r_{a}(Y)=\sum_{i=1}^{n} \xi_{i} \frac{\partial}{\partial x_{i}}$ on $U_{2}$. Then $r_{a}\left[X_{1}, Y\right]=\left[\frac{\partial}{\partial x_{1}}, \sum_{i=1}^{n} \xi_{1} \frac{\partial}{\partial x_{i}}\right]$ $=\sum_{i=1}^{n} \frac{\xi_{i} \partial}{\partial x_{1}} \frac{\partial}{\partial x_{i}}$ on $U_{2}$. Since $r_{a}\left[X_{1}, Y\right] \in \mathfrak{N}_{a}$, we have $\frac{\partial \xi_{i}}{\partial x_{1}}(a)=0$ for $i=1, \cdots, n$. Inductively we have $\frac{\partial^{k} \xi_{i}}{\partial x_{1}{ }^{k}}(a)=0$ for $i=1, \cdots, n$ and $k=1,2$, $\ldots$. Let $\alpha: \mathfrak{X}_{G}(M) \rightarrow R\left[\left[x_{1}\right]\right]$ be an $R$-module homomorphism defined by $\alpha(Z)=\sum_{k=1}^{\infty} \frac{\partial^{k} \xi_{1}}{\partial x_{1}{ }^{k}}(a) x_{1}{ }^{k}$ if $r_{a}(Z)=\sum_{i=1}^{n} \xi_{i} \frac{\partial}{\partial x_{i}}$ on $U$. Since $\alpha\left(\Im_{a}\right)=0$. 
the above map $\alpha$ induces an $R$-module homomorphism $\beta: \mathfrak{X}_{G}(M) / \mathfrak{Y}_{a} \rightarrow$ $R\left[\left[x_{1}\right]\right]$. Note that $\alpha\left(f^{j} X_{1}\right)=j ! x_{1}^{j}$ for $j=1,2, \cdots$, and $\operatorname{dim}$ (Image $\beta$ ) $=\infty$. Since $\widetilde{\Im}_{a} \supset \operatorname{Ker} \pi_{*}$, we have $\operatorname{dim} \mathfrak{X}(\bar{M}) / \bar{\Im}_{p}=\infty$.

(2) There is an index $i \in I$ such that $\&$ does not contain $J_{i}^{1}(M)$. Since $\mathfrak{Z}$ is a maximal ideal, $\mathfrak{L}+J_{i}^{1}(M)=J^{1}(M)$. Then $\mathfrak{X}_{G}(M) / \mathfrak{M}$

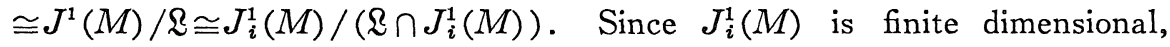
$\mathfrak{M}$ is finite codimensional. This completes the proof of Lemma 3.9.

Proposition 3.10. Let $\overline{\mathfrak{M}}$ be a maximal ideal of $\mathfrak{X}(\bar{M})$. Then

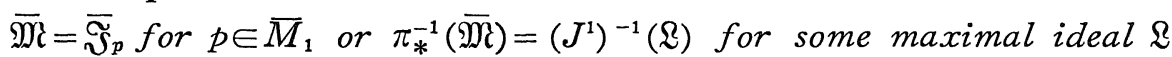
of $J^{1}(M)$.

Proposition 3.10 plays a key role to prove our theorem. We shall prove Proposition 3.10 in Section 6.

\section{$\S 4$. Stone Topology of Maximal Ideals of $\mathfrak{a}(\bar{M})$}

Let $\bar{M}^{*}$ be the set of all maximal ideals of $\mathfrak{X}(\bar{M}) . \bar{M}^{*}$ is determined by Proposition 3.10.

Definition 4. 1 (Stone topology of $\bar{M}^{*}$, cf. Pursell-Shanks [8]). The Stone topology on $\bar{M}^{*}$ is defined by closure operator CL as follows:

(1) $\mathrm{CL}(\phi)=\phi$.

(2) If $B \neq \phi$ is a subset of $\bar{M}^{*}$, then $\mathrm{CL}(B)=\left\{\mathfrak{M} \in \bar{M}^{*} ; \mathfrak{M} \supset \underset{\mathfrak{R} \in B}{\cap} \mathfrak{R}\right\}$.

Let $S\left(\bar{M}_{0}\right)$ be the set of all subsets of $\bar{M}_{0}$. Let $\tau_{M}$ (or simply $\tau$ ) be a map from $\bar{M}^{*}$ to $\bar{M}_{1} \cup S\left(\bar{M}_{0}\right)$ defined as follows:

(1) $\tau\left(\overline{\mathfrak{\Im}}_{p}\right)=p$ for $p \in \bar{M}_{1}$.

(2) If $\mathfrak{M}$ is a maximal ideal of $\mathfrak{X}(\bar{M})$ such that $J^{1}\left(\pi_{*}{ }^{-1}(\mathfrak{M})\right)$ is a maximal ideal of $J^{1}(M)$, then $\tau(\mathfrak{M})=\left\{q_{i} \in \bar{M}_{0} ; J^{1}\left(\pi_{*}{ }^{-1}(\mathfrak{M})\right)\right.$ does not contain $\left.J_{q_{i}}^{1}(\bar{M})\right\}$. (Making use of Proposition 3.5, we see that $\tau(\mathfrak{M})$ does not consist of a single point.)

For a subset $A$ of $\bar{M}$, we denote the closure of $A$ in $\bar{M}$ by $\operatorname{cl}(A)$.

Lemma 4. 2. If $\operatorname{cl}(A)$ is contained in $\bar{M}_{1}$, then $\operatorname{CL}\left(\tau^{-1}(A)\right)$ 
$=\tau^{-1}(\operatorname{cl}(A))$.

Proof. First we shall prove “ $\subset$ ”. Let $\mathfrak{M} \in \mathrm{CL}\left(\tau^{-1}(A)\right)$. Assume that $\tau(\mathfrak{M})$ is not contained in $\operatorname{cl}(A)$.

In the case $\mathfrak{M}=\overline{\mathfrak{\Im}}_{p}$ for $p \in \bar{M}_{1}:$ We can find $X \in \mathfrak{X}(\bar{M})$ such that $X_{p} \neq 0$ and $\operatorname{supp} X \cap \operatorname{cl}(A)=\phi$. Then $X \in \underset{p^{\prime} \in A}{\cap} \bar{\Im}_{p}, \subset \mathfrak{M}$, which is a contradiction to $X_{p} \neq 0$.

In the case $\pi_{*}^{-1}(\mathfrak{M})=\left(J^{1}\right)^{-1}(\mathfrak{L})$ for a maximal ideal $\mathfrak{Q}$ of $J^{1}(M)$ : There exists $Y \in \mathfrak{X}_{G}(M)$ such that $J^{1}(Y) \notin \mathbb{R}$. Let $\left\{i_{1}, \cdots, i_{k}\right\}$ be a set $\left\{i \in I ; j_{b_{i}}^{1}\left(r_{b_{i}}(Y)\right) \neq 0\right\}$. There exists $\psi \in C_{G}^{\infty}(M)$ such that $\psi=1$ on a neighborhood of $b_{i_{j}}(j=1, \cdots, k)$ and $\psi=0$ on $\pi^{-1}(\operatorname{cl}(A))$. Put $X=\psi Y$. Then $J^{1}(X)=J^{1}(Y)$, and $\pi_{*}(X) \notin \mathfrak{M}$. Moreover $\pi_{*}(X) \in \underset{p^{\prime} \in A}{\cap} \bar{\Im}_{p^{\prime}} \subset \mathfrak{M}$, which is a contradiction.

Next we shall prove "つ". Note that an ideal $\underset{\tau(\mathfrak{R}) \in A}{\cap} \mathfrak{\Re}=\underset{p^{\prime} \in A}{\bigcap} \overline{\mathfrak{\Im}}_{p}$, is contained in $\mathfrak{X}(\bar{M})_{p}$ for any $p \in \operatorname{cl}(A)$. Then $\cap \mathfrak{N}$ is contained in a maximal ideal $\overline{\widetilde{\Im}}_{p}$ and $\overline{\widetilde{\Im}}_{p} \in \operatorname{CL}\left(\tau^{-1}(A)\right)$ for any $p \in \operatorname{cl}(A)$. This completes the proof of Lemma 4.2.

If $\Phi: \mathfrak{X}(\bar{M}) \rightarrow \mathfrak{X}(\bar{N})$ is a Lie algebra isomorphism, then $\Phi^{*}: \bar{M}^{*}$ $\rightarrow \bar{N}^{*}$ is homeomorphic. Combining Lemma 3.9 and Proposition 3.10, $\Phi^{*}\left(\tau_{M}{ }^{-1}\left(\bar{M}_{1}\right)\right)=\tau_{N}{ }^{-1}\left(\bar{N}_{1}\right)$ and $\Phi^{*}\left(\tau_{M}{ }^{-1}\left(\bar{M}_{0}\right)\right)=\tau_{N}{ }^{-1}\left(\bar{N}_{0}\right)$. By Lemma 4.2, we have

Corollary 4.3. If $\Phi: \mathfrak{X}(\bar{M}) \rightarrow \mathfrak{X}(\bar{N})$ is a Lie algebra isomorphism, there exists a homeomorphism $\sigma: \bar{M}_{1} \rightarrow \bar{N}_{1}$ defined by $\sigma(p)$ $=\tau_{N}\left(\Phi^{*}\left(\overline{\widetilde{\Im}}_{p}\right)\right)$.

We shall extend the homeomorphism $\sigma: \bar{M}_{1} \rightarrow \bar{N}_{1}$ to a homeomorphism from $\bar{M}$ to $\bar{N}$.

Lemma 4. 4. Let $U$ be a neighborhood of $q \in \bar{M}_{0}$ such that $\operatorname{cl}(U) \cap \bar{M}_{0}=\{q\}$. Then $\operatorname{CL}\left(\tau^{-1}(U)\right)=\tau^{-1}(\operatorname{cl}(U))$.

The proof of Lemma 4.4 is similar to that of Lemma 4.2. 
Proposition 4.5. The map $\sigma: \bar{M}_{1} \rightarrow \bar{N}_{1}$ is extended to a homeomorphism from $\bar{M}$ to $\bar{N}$.

Proof. For $q \in \bar{M}_{0}$, let $b \in M$ such that $\pi(b)=q$. Let $V_{b}$ be a linear slice at $b$. Put $U_{q}=V_{b} / G_{b}, U_{q}^{0}=U_{q}-\{q\}$. Since $q \in \bar{M}_{0}$, it is easy to see that $U_{q}^{0}$ is connected. Note that $\underset{\tau(\mathfrak{R}) \in U_{q}{ }^{0}}{\mathfrak{R}}=\underset{\tau(\mathfrak{R}) \in U_{q}}{\bigcap}$. By Lemma 4. 4,

$$
\begin{aligned}
& \operatorname{CL}\left(\tau_{M}{ }^{-1}\left(U_{q}^{0}\right)\right)=\operatorname{CL}\left(\tau_{M}{ }^{-1}\left(U_{q}\right)\right) \\
& =\tau_{M}{ }^{-1}\left(\operatorname{cl}\left(U_{q}\right)\right)=\tau_{M}{ }^{-1}\left(\operatorname{cl}\left(U_{q}^{0}\right)\right) .
\end{aligned}
$$

Since $\Phi^{*}: \bar{M}^{*} \rightarrow \bar{N}^{*}$ is homeomorphic and since $\sigma \circ \tau_{M}=\tau_{N} \circ \Phi^{*}$,

$$
\begin{aligned}
& \operatorname{CL}\left(\tau_{N}{ }^{-1}\left(\sigma\left(U_{q}^{0}\right)\right)\right)=\operatorname{CL}\left(\Phi^{*}\left(\tau_{M}^{-1}\left(U_{q}^{0}\right)\right)\right) \\
& =\Phi^{*}\left(\operatorname{CL}\left(\tau_{M}{ }^{-1}\left(U_{q}^{0}\right)\right)\right)=\Phi^{*}\left(\tau_{M}{ }^{-1}\left(\operatorname{cl}\left(U_{q}\right)\right)\right) .
\end{aligned}
$$

There exists a maximal ideal $\mathfrak{M}_{\mathcal{C}} \in \bar{M}^{*}$ such that $\tau_{M}(\mathfrak{M})=q$. Then $\tau_{N}\left(\mathrm{CL}\left(\tau_{N}{ }^{-1}\left(\sigma\left(U_{q}^{0}\right)\right)\right)\right) \cap \bar{N}_{0}$ contains $\tau_{N}(\Phi(\mathfrak{M}))$, and, by Lemma 4.2, $\operatorname{cl}\left(\sigma\left(U_{q}^{0}\right)\right) \cap \bar{N}_{0} \neq \phi$. Since $\sigma\left(U_{q}^{0}\right)$ is connected, $\operatorname{cl}\left(\sigma\left(U_{q}^{0}\right)\right) \cap \bar{N}_{0}=\left\{q^{\prime}\right\}$ for some $q^{\prime} \in \bar{N}_{0}$. Let $\sigma(q)=q^{\prime}$ for $q \in \bar{M}_{0}$. Then it is clear that $\sigma$ : $\bar{M} \rightarrow \bar{N}$ is homeomorphic.

\section{$\S 5$. Proof of Theorem}

In this section we shall prove our theorem. Let $\Phi: \mathfrak{X}(\bar{M}) \rightarrow \mathfrak{X}(\bar{N})$ be a Lie algebra isomorphism. By Proposition 4.5, we have a homeomorphism $\sigma: \bar{M} \rightarrow \bar{N}$ such that $\sigma(p)=\tau_{N}\left(\Phi\left(\overline{\widetilde{\Im}}_{p}\right)\right)$ for $p \in \bar{M}_{1}$.

Proposition 5.1. $\sigma: \bar{M}_{1} \rightarrow \bar{N}_{1}$ is diffeomorphic.

In order to prove Proposition 5.1, we need the following lemma.

Lemma 5. 2. For $X \in X(\bar{M})$ and $p \in \bar{M}_{1}, X_{p} \neq 0$ if and only if $[X, \mathfrak{X}(\bar{M})]+\overline{\mathfrak{\Im}}_{p}=\mathfrak{X}(\bar{M})$.

Proof. Let $a \in M$ such that $\pi(a)=p$. Assume that $X_{p} \neq 0$. There exists $\widehat{X} \in \mathfrak{X}_{G}(M)$ such that $\widehat{X}_{a} \neq 0$. By the similar argument as the 
proof of Lemma 3.7 , we can prove that $\left[\widehat{X}, \mathfrak{X}_{G}(M)\right]+\mathfrak{\Im}_{a}=\mathfrak{X}_{G}(M)$, and $[X, \mathfrak{X}(\bar{M})]+\overline{\mathfrak{\Im}}_{p}=\mathfrak{X}(\bar{M})$. Conversely, suppose that $X_{p}=0$. Moreover we shall assume that $[X, \mathfrak{X}(\bar{M})]+\overline{\mathfrak{\Im}}_{p}=\mathfrak{X}(\bar{M})$. There exists $\widehat{X} \in \mathfrak{X}_{G}(M)$ such that $\pi_{*}(\widehat{X})=X$. Put $\widehat{X}^{\prime}=r_{a}(\widehat{X}) \in \Gamma_{G_{a}}\left(\tau\left(V_{a}\right)\right)$. Then $\left[\widehat{X}^{\prime}\right.$, $\left.\Gamma_{G_{a}}\left(\tau\left(V_{a}\right)\right)\right]+\mathfrak{N}_{a}=\Gamma_{G_{a}}\left(\tau\left(V_{a}\right)\right)$ and $\widehat{X}_{a}^{\prime}=0$. Let $V_{a}(0)$ and $V_{a}(1)$ be the trivial and non-trivial direct summand of the $G_{a}$-module $V_{a}$, respectively. Then $j_{a}^{1}\left(\widehat{X}^{\prime}\right)$ can be expressed as $A \oplus B$, where $A \in \mathfrak{g l}\left(V_{a}(0)\right)$ and $B \in \mathrm{gl}_{G_{a}}\left(V_{a}(1)\right)$. Since $\left[\widehat{X}^{\prime}, \Gamma_{G_{a}}\left(\tau\left(V_{a}\right)\right)\right]+\mathfrak{N}_{a}=\Gamma_{G_{a}}\left(\tau\left(V_{a}\right)\right)$, we can prove that $A$ is invertible. Then we have $\left[\widehat{X}^{\prime}, \Gamma_{G_{a}}\left(\tau\left(V_{a}\right)\right)_{0}\right]+\mathfrak{R}_{a}=\Gamma_{G_{a}}\left(\tau\left(V_{a}\right)\right)_{0}$, which implies that a linear mapping

$$
\beta: \mathfrak{g l}_{G_{a}}\left(V_{a}\right) / j_{a}^{1}\left(\mathfrak{N}_{a}\right) \rightarrow \mathfrak{g} \mathfrak{l}_{G_{a}}\left(V_{a}\right) / j_{a}^{1}\left(\mathfrak{N}_{a}\right),
$$

defined by $\beta\left(C+j_{a}^{1}\left(\mathfrak{R}_{a}\right)\right)=\left[j_{a}^{1}\left(\widehat{X}^{\prime}\right), C\right]+j_{a}^{1}\left(\mathfrak{N}_{a}\right)$ for $C \in \mathrm{gl}_{G_{a}}\left(V_{a}\right)$, is isomorphic. But this is impossible because $j_{a}^{1}\left(\widehat{X}^{\prime}\right) \in j_{a}^{1}\left(\mathfrak{N}_{a}\right)$. This completes the proof of Lemma 5.2 .

Proof of Proposition 5.1. Let $f$ be any smooth function on $\bar{N}$. Put $g=f \circ \sigma$. We have $f Y-f(\sigma(p)) Y \in \mathfrak{X}(\bar{N})_{\sigma(p)}$ for any $Y \in \mathfrak{X}(\bar{N})$, $p \in \bar{M}_{1}$ and hence, using Lemma 5.2, $\Phi^{-1}(f Y)-g(p) \Phi^{-1}(Y) \in \mathfrak{X}(\bar{M})_{p}$ for any $p \in \bar{M}_{1}$. Thus we have $\Phi^{-1}(f Y)=g \Phi^{-1}(Y)$ for any $Y \in \mathfrak{X}(\bar{N})$. For any $p \in \bar{M}_{1}$, there exist $Y \in \mathfrak{X}(\bar{N})$ and $h \in C^{\infty}(\bar{M})$ such that $\Phi^{-1}$ $(Y)(h) \neq 0$ on a neighborhood $U$ of $p$ in $\bar{M}$. Then $g=\Phi^{-1}(f Y)(h)$ $\left(\Phi^{-1}(Y)(h)\right)^{-1}$ on $U$, and $g$ is smooth on $U$. Thus $f \circ \sigma$ is smooth on $\bar{M}_{1}$ for any $f \in C^{\infty}(\bar{N})$, and $\sigma$ is smooth on $\bar{M}_{1}$. Similarly $\sigma^{-1}$ is smooth on $\bar{N}_{1}$, and Proposition 5.1 follows.

Now we shall prove that $\sigma: \bar{M} \rightarrow \bar{N}$ is diffeomorphic. By Proposition 5.1 , it is sufficient that $\sigma$ is smooth at $q \in \bar{M}_{0}$. Let $f$ be any smooth function on $\bar{N}$, and put $g=f \circ \sigma$. As in the proof of Proposition 5.1, $g \Phi^{-1}(Y)=\Phi^{-1}(f Y)$ for any $Y \in \mathfrak{X}(\bar{N})$. Since $\Phi$ is a Lie algebra isomorphism, $g X(h) \in C^{\infty}(\bar{M})$ for any $X \in \mathfrak{X}(\bar{M}), h \in C^{\infty}(\bar{M})$. Let $b$ be a point of $M$ such that $\pi(b)=q$, and let $V$ be a linear slice at $b$. Let $H$ be the isotropy subgroup at $b$, and put $\bar{V}=V / H$. It suffices to prove the following:

Proposition 5.3. Let $g$ be a continuous function on $\bar{V}$ such that 
$g X(h) \in C^{\infty}(\bar{V})$ for any $X \in \Gamma(\tau(\bar{V})), h \in C^{\infty}(\bar{V})$. Then $g$ is a smooth function.

Let $\left\{\theta_{1}, \cdots, \theta_{s}\right\}$ be a minimal set of homogeneous generators of $R[V]_{0}^{H}$ (see Davis [3], Lemma 4.6). Let $\left\{x_{1}, \cdots, x_{n}\right\}$ be a canonical coordinate of $V$ such that $H$ acts orthogonally on this coordinate. Since $q \in \bar{M}_{0}$, $\Gamma(\tau(\bar{V}))=\Gamma(\tau(\bar{V}))_{0}$. It is easy to see that $\operatorname{deg} \theta_{i}>1$ for $i=1, \cdots, s$. Then we can assume $\theta_{1}=x_{1}{ }^{2}+\cdots+x_{n}{ }^{2}$. Let $X$ be a radial vector field $\sum_{i=1}^{n} x_{i} \frac{\partial}{\partial x_{i}}$. Then $X\left(\theta_{i}\right)=\left(\operatorname{deg} \theta_{i}\right) \theta_{i}$ for $i=1, \cdots, s$, and Proposition 5.3 follows from the following:

Proposition 5.4. Let $g$ be an H-invariant continuous function on $V$ such that $\theta_{i} g \in C_{H}^{\infty}(V)$ for $i=1, \cdots, s$. Then $g$ is an $H$-invariant smooth function.

Proof. Since $\theta_{1} g \in C_{H}^{\infty}(V)$, it is sufficient to prove that $g$ is smooth at 0 . Put $g_{1}=\theta_{1} g \in C_{H}^{\infty}(V)$. First we consider the case $s=1$. In this case $\bar{V}$ is a half line $R_{+}$, it follows from Theorem 1.2 that $\bar{g}_{1}=x \bar{g}$ is a smooth function on $R_{+}$, where $\bar{g}_{1}$ and $\bar{g}$ are functions on $R_{+}$such that $g_{1}=\bar{g}_{1} \circ \theta_{1}$ and $g=\bar{g} \circ \theta_{1}$, respectively. By Koriyama [5] Lemma 6.2, $\bar{g} \in$ $C^{\infty}\left(R_{+}\right)$, and hence $g \in C_{H}^{\infty}(V)$.

Now we consider the case $s \geqq 2$. Put $R[V]_{i}^{H}=\left\{h \in R[V]^{H} ; \operatorname{deg} h\right.$ $=i\}$. From Taylor's formula, for an integer $m \geqq 2$, there exist $P_{m}(x)$ $\in \sum_{2 \leqq i \leqq m} R[V]_{i}^{H}$ and $R_{m}(x) \in \sum_{|I|=m+1} x^{I} C^{\infty}(V)$ such that $g_{1}(x)=P_{m}(x)$ $+R_{m}(x)$, where $x^{I}=x_{1}^{i_{1}} \cdots x_{n}{ }^{i_{n}}$ and $|I|=i_{1}+\cdots+i_{n}$. Put $g_{2}=\theta_{2} \cdot g_{1}, k=$ $\operatorname{deg} \theta_{2}+m$. Then $\theta_{2} P_{m} \in \sum_{2 \leqq i \leqq k} R[V]_{i}^{H}$ and $\theta_{2} R_{m}(x) \in \sum_{|I|=k+1} x^{I} C^{\infty}(V)$. Let $\Delta=\sum_{i=1}^{n} \frac{\partial^{2}}{\partial x_{i}{ }^{2}}$ be the Laplacian, and put $H^{k}(V)=\left\{f \in R[V]_{k} ; \Delta f\right.$ $=0\}$. Here we need the following result:

Theorem 5.5 (cf. [13] § 14).

$$
R[V]_{i}=\theta_{1} R[V]_{i-2} \oplus H^{i}(V) \text { for an integer } i \geqq 2 \text {. }
$$

From Theorem 5.5, there exist $Q_{m} \in \sum_{i \leqq k-2} R[V]_{i}$ and $T_{m} \in$ $\sum_{i \leqq k} H^{i}(V)$ such that $\theta_{2} P_{m}=\theta_{1} Q_{m}+T_{m}$. 
Proposition 5. 6. $T_{m}=0 \quad(m=2,3, \cdots)$.

Proof. It is easy to see the following:

(1) For $f \in H^{l}(V), \Delta^{p}\left(f / \theta_{1}\right)=N(p, l) f / \theta_{1}{ }^{p+1}$, where $N(p, l)=$ $-\left[4 p l+2 p n-4\left(4(p-1)^{2}+12(p-1)+8\right)\right]$.

(2) For $F_{I} \in C^{\infty}(V)$ with $|I|=k+1$, put $F(x)=\sum_{|I|=k+1} F_{I}(x) x^{I}$. Then we have $\Delta^{p}\left(F / \theta_{1}\right)=\sum_{q=1}^{p+1} F^{p, q} / \theta_{1}^{q}$ for some $F^{p, q}(x) \in \sum_{|I|=k+2 q-2 p-1}$ $x^{I} C^{\infty}(V)$.

Now we assume that $T_{m}=\sum_{i=d}^{k} T_{m}{ }^{i}$ such that $T_{m}{ }^{i} \in H^{i}(V)$ and $T_{m}{ }^{d}$ $\neq 0$. Let $d_{1}$ be an integer such that $d=2 d_{1}+1$ or $2 d_{1}+2$. To see Proposition 5.5 , we can assume that $k$ is an even integer, and hence $k \geqq 2 d_{1}$ +2. By the definitions of $g_{1}$ and $g_{2}, \theta_{2} g=g_{2} / \theta_{1}=Q_{m}+T_{m} / \theta_{1}+\theta_{2} R_{m} / \theta_{1}$. Since $\theta_{2} g$ is a smooth map, $T_{m} / \theta_{1}+\theta_{2} R_{m} / \theta_{1}$ is also a smooth map.

Put $F=\theta_{2} R_{m}$. Applying (2), we have $\Delta^{d_{1}}\left(F / \theta_{1}\right)=\sum_{q=1}^{d_{1}+1} F^{d_{1}, q} / \theta_{1}{ }^{q}$ for some $F^{d_{1}, q}(x) \in \sum_{|I|=k+2 q-2 d_{1}-1} \in x^{I} C^{\infty}(V)$. Let $a=\left(a_{1}, \cdots, a_{n}\right) \in V$ with $a$ $\neq 0$ and $\xi$ be a positive real number. Since $k-2 d_{1}-1>0$, it is easy to see that

$$
\left.\lim _{\xi \rightarrow 0} \Delta^{d_{1}}\left(F / \theta_{1}\right)\right|_{x=\xi a}=0 .
$$

It follows from (1) that

$$
\left.\lim _{\xi \rightarrow 0} \Delta^{d_{1}}\left(T_{m}^{i} / \theta_{1}\right)\right|_{x=\xi a}=0 \quad \text { for } \quad i \geqq 2 d_{1}+2 .
$$

We can write

$$
\begin{array}{lll}
T_{m}{ }^{2 d_{1}+1}(x)=\sum_{|I|=2 d_{1}+1} \lambda_{I} x^{I} & \text { for } & \lambda_{I} \in R, \\
T_{m}{ }^{2 d_{1}+2}(x)=\sum_{|J|=2 d_{2}+2} \mu_{J} x^{J} & \text { for } & \mu_{J} \in R,
\end{array}
$$

Then $\left.d^{d 1}\left(T_{m}{ }^{2 d_{1}+1}(x) / \theta_{1}+T_{m}{ }^{2 d_{1}+2}(x) / \theta_{1}\right)\right|_{x=\xi a}=\left(N\left(d_{1}, 2 d_{1}+1\right) \sum \lambda_{I} a_{I}\right) /(\xi$ $\left.\|a\|^{2 d_{1}+2}\right)+\left(N\left(d_{1}, 2 d_{1}+2\right) \sum \mu_{J} a_{J}\right) /\|a\|^{2 d_{1}+2}$. Note that $N\left(d_{1}, 2 d_{1}+1\right) \neq 0$, $N\left(d_{1}, 2 d_{1}+2\right) \neq 0$. Since $T_{m} / \theta_{1}+F / \theta_{1}$ is smooth, it follows from (3) that

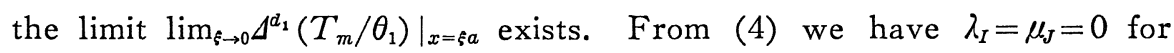
any $I, J$. Then $T_{m}{ }^{d}=0$, which is a contradiction. Therefore $T_{m}=0$.

Proof of Proposition 5.4 continued. From Proposition 5.6, we have $\theta_{2} P_{m}=\theta_{1} Q_{m}$. Since $\left\{\theta_{1}, \cdots, \theta_{s}\right\}$ is a minimal set of generators, there exists an $H$-invariant polynomial $P_{m}{ }^{\prime}$ such that $P_{m}=\theta_{1} P_{m}{ }^{\prime}$. Then $g=g_{1} / \theta_{1}$ $=P_{m}{ }^{\prime}+R_{m} / \theta_{1}$ for $R_{m}(x) \sum_{|I|=m+1} x^{I} C^{\infty}(V)(m=2,3, \cdots)$, and $g$ is a smooth 
map. This completes the proof of Proposition 5.4.

To complete the proof of our theorem, we shall prove that $\Phi=\sigma_{*}$. Similar way as in the proof of Proposition 5.1, for any $f \in C^{\infty}(M)$, $X \in \mathfrak{X}(\bar{M})$, we have $\Phi(f X)=\left(f \circ \sigma^{-1}\right) \Phi(X)$. Then $\Phi(X)\left(f \circ \sigma^{-1}\right) \Phi(X)$ $=\Phi(X(f) X)=\Phi([X, f X])=[\Phi(X), \Phi(f X)]=\left[\Phi(X),\left(f \circ \sigma^{-1}\right) \Phi(X)\right]$ $=\Phi(X)\left(f \circ \sigma^{-1}\right) \Phi(X)$. Hence $\Phi(X)\left(f \circ \sigma^{-1}\right) \Phi(X)=\Phi(X)\left(f \circ \sigma^{-1}\right) \Phi(X)$, and we see $\Phi(X)\left(f \circ \sigma^{-1}\right)=X(f) \circ \sigma^{-1}$. Then, for any $g \in C^{\infty}(\bar{N})$, $X \in X(\bar{M}), \Phi(X)(g)=X(g \circ \sigma) \circ \sigma^{-1}=\sigma_{*}(X)(g)$, and hence $\Phi=\sigma_{*}$. This completes the proof of our theorem.

Remark. We can prove that $\sigma$ is strata preserving.

\section{§ 6. Proof of Proposition 3.10}

In this section we shall prove Proposition 3.10. The proof is parallel to those of Pursell-Shanks [8] and Koriyama [5]. We start with some lemmas.

Lemma 6. 1 (Sternberg [12]). Let $X$ be a radial vector field on $R^{n}$ defined by $X=\sum_{i=1}^{n} x_{i} \frac{\partial}{\partial x_{i}}$. Let $Y$ be a smooth vector field on $R^{n}$ such that $j_{0}^{1}(X)=j_{0}^{1}(Y)$. Then there exists a local coordinate system $\left(y_{1}, \cdots, y_{n}\right)$ defined on a neighborhood $U$ of 0 such that $Y=$ $\sum_{i=1}^{n} y_{i} \frac{\partial}{\partial y_{i}}$ on $U$.

Lemma 6.2. Let $\bar{M}$ be an ideal of $\mathfrak{X}(\bar{M})$ such that for any $p \in \bar{M}_{1}$ there exists $\bar{Y} \in \overline{\mathfrak{M}}$ such that $\bar{Y}_{p} \neq 0$. Then $\overline{\mathfrak{M}}$ contains an ideal $\bar{\Im}_{1}=\left\{\bar{X} \in \mathfrak{X}(\bar{M})\right.$; supp $\left.\bar{X} \subset \bar{M}_{1}\right\}$.

Proof. Put $\mathfrak{M}=\pi_{*}{ }^{-1}(\overline{\mathfrak{M}}), \mathfrak{\Im}_{1}=\pi_{*}{ }^{-1}\left(\overline{\mathfrak{\Im}}_{1}\right)$. We shall prove that $\mathfrak{M}$ contains $\widetilde{\Im}_{1}$. Similarly as in the proof of Lemma 3.9 , for any point $a$ $\in \pi^{-1}\left(\bar{M}_{1}\right)$ there exist a local coordinate system $\left(x_{1}, \cdots, x_{n}\right)$ around a $G_{a}$-invariant neighborhood $U_{a}$ of $a$ in $V_{a}$ and $Y \in \mathfrak{M}$ such that $r_{a}(Y)$ $=\frac{\partial}{\partial x_{1}}$ on $U_{a} . \quad x_{1}$ is extended to a $G$-invariant smooth function $f$ on $M$. 
Put $Y_{1}=Y-r_{a}(Y)$ on $V_{a}$. Then $\pi_{*}\left(Y_{1}\right)=0$ on $\bar{V}_{a}$. Using a partition of unity, we can find $Y_{2} \in \operatorname{Ker} \pi_{*} \subset \mathfrak{M}$ such that $Y_{2}=Y_{1}$ on $U_{a}$. Put $Y_{3}=Y-Y_{2}$. Then $Y_{3}=r_{a}(Y)=\frac{\partial}{\partial x_{1}}$ on $U_{a}$ and $Y_{3} \in \mathfrak{M}$.

Let $X \in \mathfrak{\Im}_{1}$. To prove $X \in \mathfrak{M}$ we can assume that supp $X$ is contained in $G \times{ }_{G_{a}} V_{a}$ by using arguments of invariant partition of unity. Moreover, we can assume that $r_{a}(X)=X$ on $U_{a}$ since $\operatorname{Ker} \pi_{*}$ is contained in $\mathfrak{M}$. Put $X=\sum_{i=1}^{n} \xi_{i} \frac{\partial}{\partial x_{i}}$ on $U_{a}$. Since $x_{1}$ is a $G_{a}$-invariant function, we see that $\xi_{1}$ is a $G_{a}$-invariant function. Similarly as in the proof of Koriyama [5] Lemma 2.13, we can prove that $X$ is an element of $\mathfrak{M}$. This completes the proof of Lemma 6.2.

$$
\text { Put } \mathfrak{X}_{G}(M)_{0}^{\infty}=\bigcap_{i \in I} r_{b_{i}}^{-1}\left(\Gamma_{G_{b_{i}}}\left(\tau\left(V_{b_{i}}\right)\right)_{0}^{\infty}\right)
$$

Lemma 6. 3. Let $\overline{\mathfrak{M}}$ be an ideal of $\mathfrak{X}(\bar{M})$ such that $J^{1}\left(\pi_{*}{ }^{-1}(\overline{\mathfrak{M}})\right)$ $=J^{1}(M)$ and for any $p \in \bar{M}_{1}$ there exists an element $\bar{Y} \in \bar{M}$ such that $\bar{Y}_{p} \neq 0$. Then an ideal $\mathfrak{M}=\pi_{*}{ }^{-1}(\overline{\mathfrak{M}})$ of $\mathfrak{X}_{G}(M)$ contains an ideal $\mathfrak{X}_{\theta}(M)_{0}^{\infty}$.

Proof. Since $J^{1}(\mathfrak{M})=J^{1}(M)$, there exists an element $X \in \mathfrak{M}$ such that $j_{b_{i}}^{1}\left(r_{b_{i}}(X)\right)=j_{b_{i}}^{1}\left(\Re_{i}\right)$. Here $\Re_{i}$ is a radial vector field on $V_{b_{i}}$ defined by $\Re_{i}=\sum_{j=1}^{n} y_{j} \frac{\partial}{\partial y_{j}}$ on $V_{b_{i}}$, where $\left\{y_{1}, \cdots, y_{n}\right\}$ is a canonical coordinate of $V_{b_{i}}$. Since $\mathfrak{M}$ contains $\operatorname{Ker} \pi_{*}$, we can assume that $X=\sum_{j=1}^{n} y_{j} \frac{\partial}{\partial y_{j}}$ on $U_{i}$.

As in the proof of Koriyama [5] Lemma 2.13, for any element $Z \in \mathfrak{X}_{G}(M)_{0}{ }^{\infty}$, there exist vector fields $Y_{i}, i \in I$, such that $\left[X, Y_{i}\right]=r_{b_{i}}(Z)$ on a $G_{b_{i}}$-invariant neighborhood $W_{i} \subset U_{i}$ of $b_{i}$ and supp $Y_{i} \subset U_{i}$. Put $\widetilde{Y}_{i}=\int_{\sigma_{b_{i}}} g_{*} Y_{i} d g \in \Gamma_{G_{b_{i}}}\left(\tau\left(V_{b_{i}}\right)\right)$. By Lemma 3.6, we have $\left[X, \widetilde{Y}_{i}\right]=r_{b_{i}}(Z)$ on $W_{i}$. By Lemma 3.1 (1), we can assume $\widetilde{Y}_{i} \in \mathfrak{X}_{G}(M)$ and supp $\widetilde{Y}_{i}$ is contained in $G \times{ }_{b_{b_{i}}} U_{i}$. Since supp $Z$ is compact, there is a finite index set $\left\{i_{1}, \cdots, i_{k}\right\} \subset I$ such that $\operatorname{supp} Z \cap G \times \times_{b_{b_{i}}} V_{b_{i_{j}}} \neq \varnothing$. Put $\widetilde{Y}=\widetilde{Y}_{i_{1}}+\cdots$ $+\widetilde{Y}_{i_{k}}$. Since Ker $\pi_{*}$ is contained in $\mathfrak{M}$, there exists an element $Z_{0} \in \mathfrak{M}$ such that $Z_{0}=Z-r_{b_{i}}(Z)$ on $W_{i,}$ for $j=1, \cdots, k$. Then $Z_{1}=Z-Z_{0}-[X, \widetilde{Y}]$ is an element of $\mathfrak{\Im}_{1}$ in Lemma 6.2 , and $Z_{1} \in \mathfrak{M}$. Thus we have $Z \in \mathfrak{M}$, 
and this completes the proof of Lemma 6.3.

Lemma 6.4. Let $\overline{\mathfrak{M}}$ be a maximal ideal of $\mathfrak{X}(\bar{M})$ such that for any $p \in \bar{M}_{1}$ there exists an element $\bar{Y} \in \overline{\mathfrak{M}}$ such that $\bar{Y}_{p} \neq 0$. Then $J^{1}(\mathfrak{M})$ is a maximal ideal of $J^{1}(M)$, where $\mathfrak{M}=\pi_{*}^{-1}(\overline{\mathfrak{M}})$.

Proof. Suppose $J^{1}(\mathfrak{M})=J^{1}(M)$. We shall prove that $\operatorname{Ker} J^{1}$ is contained in $\mathfrak{M}$. By Lemma 6.2, it is enough to prove that an element $Z \in \operatorname{Ker} J^{1}$ satisfying supp $Z \subset G \times{ }_{a_{b_{i}}} V_{b_{i}}$ is an element of $\mathfrak{M}$. Since Ker $\pi_{*}$ is contained in $\mathfrak{M}$, we can assume $Z=r_{b_{i}}(Z)$ on $V_{b_{i}}$. As in the proof of Lemma 6.3 , there exist $X \in \mathfrak{M}$ and a local coordinate system $\left(x_{1}, \cdots, x_{n}\right)$ defined on a $G_{b_{i}}$-invariant neighborhood $U_{i}$ of $b_{i}$ in $V_{b_{i}}$ such that $X=\sum_{j=1}^{n} x_{i} \frac{\partial}{\partial x_{i}}$ on $U_{i}$.

From the proof of Koriyama [5] Lemma 2.10, there exists a smooth vector field $Y$ on $V_{b_{i}}$ such that $Z_{1}=Z-[X, Y] \in \Gamma\left(\tau\left(V_{b_{i}}\right)\right)_{0}{ }^{\infty}$. Put $\widetilde{Y}=\int_{\sigma_{b_{i}}} g_{*} Y d g$. Then $Z-[X, \widetilde{Y}]=\int_{G_{b_{i}}} g_{*} Z_{1} d g \in \Gamma_{G_{b_{i}}}\left(\tau\left(V_{b_{i}}\right)\right)_{0}^{\infty}$. By Lemma 3.1 (1), we can assume $\widetilde{Y} \in \mathfrak{X}_{G}(M)$, and $Z-[X, \widetilde{Y}] \in \mathfrak{X}_{G}(M)_{0}{ }^{\infty}$. Then it follows from Lemma 6.3 that $Z \in \mathfrak{M}$. Thus $\operatorname{Ker} J^{1}$ is contained in $\mathfrak{M}$. Since $J^{1}(\mathfrak{M})=J^{1}(M), \mathfrak{M}=\mathfrak{X}_{G}(M)$. This is a contradiction, and this completes the proof of Lemma 6.4.

Proof of Proposition 3.10. Let $\overline{\mathfrak{M}}$ be a maximal ideal of $\mathfrak{X}(\bar{M})$. If there exists a point $p \in \bar{M}_{1}$ such that $\overline{\mathfrak{M}}$ is contained in $\mathfrak{X}(\bar{M})_{p}$, then $\overline{\mathfrak{M}}=\overline{\mathfrak{\Im}}_{p}$. Suppose for any point $p \in \bar{M}_{1}$ there exists an element $\bar{X} \in \overline{\mathfrak{M}}$ such that $\bar{X}_{p} \neq 0$. By Lemma $6.4, J^{1}\left(\pi_{*}^{-1}(\overline{\mathfrak{M}})\right)$ is a maximal ideal of $J^{1}(M)$. This completes the proof of Proposition 3.10.

\section{References}

[1] Amemiya, I., Lie algebra of vector fields and complex structure., J. Math. Soc. Japan, 27 (1975), 545-549.

[2] Bierstone, E., Lifting isotopies from orbit spaces, Topology, 14 (1975), 245-252.

[3] Davis, M., Smooth G-manifolds as collections of fibre bundles, Pacific J. Math., 77 (1978), 315-363.

[4] Fukui, K., Pursell-Shanks type theorem for free G-manifolds, Publ. Res. Inst. Math. Sci., 17 (1981), 249-265.

[5] Koriyama, A., On Lie algebras of vector fields with invariant submanifolds, Nagoya J. Math., 55 (1974), 91-110. 
[6] Omori, H., Infinite dimensional Lie transformation groups, Lecture Notes in Math., 427 Springer-Verlag, 1976.

[7] Poénaru, V., Singularité $C^{\infty}$ en présence de symétrie, Lecture Notes in Math., 510 Springer-Verlag, 1976.

[8] Pursell, L. E. and Shanks, M. E., The Lie algebra of a smooth manifold, Proc. Amer. Math. Soc., 5 (1954), 468-472.

[9] Schwarz, G. W., Smooth invariant functions under the action of a compact Lie group, Topology, 14 (1975), 63-68.

[10] , Covering smooth homotopies of orbit spaces, Bull. Amer. Math. Soc., 83 (1977), 1028-1030.

[11] - Lifting smooth homotopies of orbit spaces, Inst. Hautes Etudes Sci. Publ. Math., 51 (1980), 37-135.

[12] Sternberg, S., Local contractions and a theorem of Poincaré, Amer. J. Math., 79 (1957), 809-824.

[13] Takeuchi, M., Modern Spherical functions, Iwanami-Shoten, 1975. (in Japanese)

[14] Weyl, H., The Classical Groups, 2nd edition, Princeton, Univ. Press, Princeton, New Jersey, 1973. 